\title{
Understanding the Differences Between Gastroparesis and Gastroparesis-Like Syndrome: Filling a GaPing Hole?
}

\author{
Jonathan Gotfried $^{1} \cdot$ Ron Schey $^{1}$
}

Published online: 3 August 2017

(C) Springer Science+Business Media, LLC 2017

\section{Background}

The terms gastroparesis (GP) and gastroparesis-like syndrome (GLS) are used to diagnose patients experiencing similar symptoms of nausea, vomiting, and postprandial abdominal pain with GP defined as this symptom complex accompanied by delayed gastric emptying (GE). A current conundrum is why similar symptoms are present in these two groups despite differing gastric motor function. Historically, studies have focused on interstitial cells of Cajal (ICC) networks and collagen fibrosis in the gastric antrum, although their results do not explain the development of delayed gastric emptying (GE) in some patients and not in others. Growing interest in the pylorus stems from attempts to determine whether aberrant pyloric function is the mechanism that delays GE in GP and not in GLS patients, despite both groups experiencing similar symptoms. Previously, authors have suggested that GP and GLS exist along a continuum of the same pathophysiologic process of a complex interplay of inflammation, fibrosis, and neural network changes that alter gastric motility and eventually lead to delayed GE [1]. As such, the contribution of each of these physiologic derangements to the development of symptoms and, in some patients, delayed GE is unknown. A better understanding of the pathophysiologic mechanisms that lead to the unique clinical manifestations of GP and GLS is thus essential to developing novel therapies [2].

Ron Schey

Ron.schey@tuhs.temple.edu

1 Neurogastroenterology and Esophageal Disorders Program, Section of Gastroenterology, Lewis Katz School of Medicine, Temple University and the Temple University Health System, Philadelphia, PA, USA
There is still debate over the definition of GLS and its relationship to GP. GE as assessed by nuclear scintigraphy may change over time and is influenced by systemic processes and medications. Moreover, patients in studies of GP and GLS are heterogeneous, manifesting the disease with varying degrees of symptoms, comorbidities, and response to medications, complicating study of this group. The recent study by Bashashati et al. [3] published in this month's issue of Digestive Diseases and Sciences is noteworthy for its attempt to characterize pyloric changes in patients with GP or GLS by determining the amount of pyloric collagen deposition and ICC loss. The study compares GP and GLS patients; it is important to recognize that the data represent a single point on a longitudinal scale of symptomatology and disease progression as assessed by gastric emptying nuclear scintigraphy, influencing any conclusions that may be derived from the data.

\section{Pylorus Pathology Contribution to Delayed Gastric Emptying}

The degree to which the pylorus contributes to symptoms and delayed GE is unknown. Several techniques that have been employed to decrease pylorus resistance have had varying results in symptom improvement and GE. In a retrospective analysis of prospectively collected data, pyloroplasty alone in GP patients was associated with overall improvement in symptoms and improved GE in $83 \%$ of patients surveyed [4]. Yet, other studies have described limited success rates, reporting only up to $30 \%$ of subjects with normalization of (GE) and some improvement in symptoms following pyloroplasty without gastric stimulator placement. Although gastric per oral endoscopic myotomy (G-POEM) is an alternative, less invasive 
method of disrupting the pylorus, no prospective data are available on its use in GP. To date, one large, multicenter retrospective analysis of 30 patients with various forms of GP found an $86 \%$ clinical response after G-POEM, a finding that must be confirmed by the collection of longterm data and by the conduct of randomized trials [5]. Other methods to relieve pyloric resistance include botulinum toxin injection and self-expanding covered metallic stents that decrease GP symptoms [6]. Studies of this type should be interpreted with the understanding that a wide range of symptom response to pyloric interventions has been reported across studies using the same technique. These studies suggest that although pyloric dysfunction contributes to delayed GE, there are other mechanisms that likely contribute to the development of GP.

\section{Interstitial Cells of Cajal}

Previous studies in rodent models have suggested the lack of ICC populations in the pylorus allows for distinct antral and duodenal motor activity whereby duodenal distension from deposited gastric contents stimulates aboral duodenal contractions that enable independent contractions at appropriate intervals [7]. Nonetheless, ICC populations are essential for motility, as demonstrated by studies of the contribution of ICC to pyloric stenosis and hypertrophy in pediatric patients [8]. Significant heterogeneity exists among studies examining the number of ICC in the gastric antrum. Few studies have investigated ICC networks in the pylorus, with only one other human study to date (conducted by the current study's author) [9-11].

Prior studies have highlighted the importance of ICC loss in GP. In a prospective study at our institution assessing 145 patients with refractory diabetic or idiopathic GP undergoing GES placement, the global response to GES was inversely related to the presence of ganglia [11]. As the numbers of ICC in the gastric antrum were the same in GP and GLS patients in the current study, one may conclude that there may not be a clinically meaningful difference even with ICC changes in the pylorus in GP patients. In rat pylorus, ICC networks are separate and distinct throughout the upper GI tract, independent of afferent innervation. Further investigation into central and enteric nervous system stimulation and its contribution to pylorus function is also needed when considering the global effect of pylorus dysfunction on GE [12]. As suggested by Patterson et al., ICC may mediate some of the effects of cholecystokinin on pyloric sphincter pressure. Further studies on the potential contributions of ICC to the coordination of gastroduodenal motility are needed.

This study and previous studies by the same authors have found similar ICC loss in gastric antrum in patients with GP and GLS [9]. As pointed out by Wang et al., an older study in dogs demonstrated antral contractile forces open the pylorus $[13,14]$. The current study finding of reduced antral smooth muscle ICC in both GP and GLS cannot be extrapolated to support this finding.

\section{Degree of Fibrosis}

Although the study found the same level of fibrosis in the antrum of GP and GLS, significantly more fibrosis is present in the pylorus in GP patients, a finding that suggests that fibrosis in the pylorus may be the principal contributor to the pathogenesis of delayed GE in GP patients. Other studies have shown the degree of stretch of smooth muscle may influence the contractile forces generated in the antrum of rats [15]. Whether or not fibrosis is a useful surrogate for clinical pyloric dysfunction is unknown as there are no data correlating the presence or degree of fibrosis with meaningful clinical standards or metrics of gastric emptying. As suggested by the authors, further studies into the clinical consequences of pyloric fibrosis are needed, potentially with real-time measures such as EndoFLIP (endoluminal functional lumen imaging probe), a through-the-scope device used to measure distensibility of gastrointestinal organs by simultaneously measuring the area across the inside of a given organ (e.g., pylorus or lower esophageal sphincter) and pressure inside that organ.

In a previous study comparing idiopathic- and diabeticGP patients with healthy controls, surgical biopsy documented loss of ICC in $83 \%$ of gastroparesis patients though only $3 / 40$ total GP patients had fibrosis on antral biopsies [10]. Considering the low amount of fibrosis reported by other studies such as by Grover et al. [10], the authors' findings suggesting a correlation of antral fibrosis and pyloric fibrosis are difficult to interpret given the data of patients with GP where a minority have fibrosis present yet most have symptoms of gastroparesis. Faussone-Pelligrini et al. [16] reported ultrastructural differences between idiopathic and diabetic GP with more severe changes present in idiopathic GP. Further analysis by the authors by subgroup (idiopathic vs diabetic GP vs GLS) is needed as the more severe IGP may be overrepresented in a smaller population. Interestingly, in a study from our institution, despite the same level of fibrosis in DGP and in IGP, patients undergoing GES placement experienced improved symptoms in the DGP but not IGP suggesting other unknown mechanisms contributing to their current disease state [11]. Overall though, antral fibrosis was predictive of poor response to GES compared to patients without significant fibrosis. Nevertheless, based on the findings in the current study, evaluation of the presence of fibrosis in the pylorus should be considered an important diagnostic tool 
when characterizing and treating patients with GP and GLS.

\section{Future Directions}

Characterizing pyloric fibrosis and ICC populations in GP and GLS disease states is important and has not been adequately evaluated in previous studies. Whether or not the degree of pylorus dysfunction in these states is related to these measures or others (i.e., antral contraction effect on pylorus or gastric dysrhythmias) remains to be seen. As the authors point out, GP and GLS may exist on a continuum; as an example, these diseases are frequently reclassified when assessed at time points from baseline48 weeks after reimaging [2]. Further studies are needed to understand the pathophysiologic basis of GP and GLS since there is substantial morbidity in this patient cohort and since interventions may be invasive and potentially carry significant risk including postoperative mortality [11]. Understanding which histopathologic findings, not only in the gastric antrum but also in the gastric pylorus, of the GP and GLS patient population correspond to clinically meaningful outcomes is of importance to inform the development of safer and more effective interventions.

\section{Key Messages}

- Both gastroparesis and gastroparesis-like syndrome have similar symptoms. They are differentiated by the presence of delayed gastric emptying in gastroparesis.

- Both gastroparesis and gastroparesis-like syndrome can have reduced antral interstitial cells of Cajal.

- Gastroparesis, but not gastroparesis-like syndrome, has decreased interstitial cells of Cajal and fibrosis in the pylorus.

- The pathologic abnormalities in the pylorus involving ICC and fibrosis may decrease pyloric sphincter function, impairing gastric emptying.

- Focusing on ICC and fibrosis in the pylorus in addition to the gastric antrum is important to the design of future studies.

\section{References}

1. Harer KN, Pasricha PJ. Chronic unexplained nausea and vomiting or gastric neuromuscular dysfunction (GND)? An update on nomenclature, pathophysiology and treatment, and relationship to gastroparesis. Curr Treat Opt Gastroenterol. 2016;14:410-419.

2. Anaparthy R, Pehlivanov N, Grady J, Yimei H, Pasricha PJ. Gastroparesis and gastroparesis-like syndrome: response to therapy and its predictors. Dig Dis Sci. 2009;54:1003-1010. doi:10. 1007/s10620-009-0717-4.

3. Bashashati M, Moraveji S, Torabi A, et al. Pathological findings of the antral and pyloric smooth muscle in patients with gastroparesis-like syndrome compared to gastroparesis: similarities and differences. Dig Dis Sci. (Epub ahead of print). doi:10.1007/ s10620-017-4629-4.

4. Hibbard ML, Dunst CM, Swanström LL. Laparoscopic and endoscopic pyloroplasty for gastroparesis results in sustained symptom improvement. J Gastrointest Surg. 2011;15:1513-1519.

5. Khashab MA, Ngamruengphong S, Carr-Locke D, et al. Gastric per-oral endoscopic myotomy for refractory gastroparesis: results from the first multicenter study on endoscopic pyloromyotomy (with video). Gastrointest Endosc. 2017;85:123-128.

6. Reddymasu SC, Singh S, Sankula R, Lavenbarg TA, Olyaee M, McCallum RW. Endoscopic pyloric injection of botulinum toxinA for the treatment of postvagotomy gastroparesis. Am J Med Sci. 2009;337:161-164.

7. Wang XY, Lammers WJ, Bercik P, Huizinga JD. Lack of pyloric interstitial cells of cajal explains distinct peristaltic motor patterns in stomach and small intestine. Am J Physiol Gastrointest Liver Physiol. 2005;289:G539-G549.

8. Vanderwinden J, Liu H, De Laet M, Vanderhaeghen J. Study of the interstitial cells of cajal in infantile hypertrophic pyloric stenosis. Gastroenterology. 1996;111:279-288.

9. Moraveji S, Bashashati M, Elhanafi S, et al. Depleted interstitial cells of cajal and fibrosis in the pylorus: novel features of gastroparesis. Neurogastroenterol Motil. 2016;28:1048-1054.

10. Grover M, Farrugia G, Lurken MS, et al. Cellular changes in diabetic and idiopathic gastroparesis. Gastroenterology. 2011;140: 1575.e8-1585.e8.

11. Heckert J, Thomas R, Parkman H. Gastric neuromuscular histology in patients with refractory gastroparesis: relationships to etiology, gastric emptying, and response to gastric electric stimulation. Neurogastroenterol Motil. 2017;29:e13068.

12. Patterson LM, Zheng H, Ward SM, Berthoud H. Immunohistochemical identification of cholecystokinin A receptors on interstitial cells of cajal, smooth muscle, and enteric neurons in rat pylorus. Cell Tissue Res. 2001;305:11-23.

13. Prove J, Ehrlein HJ. Motor function of gastric antrum and pylorus for evacuation of low and high viscosity meals in dogs. Gut. 1982;23:150-156.

14. Wang X, Huizinga J, Diamond J, Liu L. Loss of intramuscular and submuscular interstitial cells of cajal and associated enteric nerves is related to decreased gastric emptying in streptozotocininduced diabetes. Neurogastroenterol Motil. 2009;21:1095-e92.

15. Zhang R, Wang X, Chen D, Huizinga J. Role of interstitial cells of cajal in the generation and modulation of motor activity induced by cholinergic neurotransmission in the stomach. $\mathrm{Neu}$ rogastroenterol Motil. 2011;23:e356-e371.

16. Faussone-Pellegrini MS, Grover M, Pasricha PJ, et al. Ultrastructural differences between diabetic and idiopathic gastroparesis. J Cell Mol Med. 2012;16:1573-1581. 